DOCTRINA

\title{
Filtro burbuja: ¿Cuál es el costo de la personalización digital?
}

\author{
The bubble filter: What is the cost of digital personalization?
}

\author{
Fernando Bajaña Tovar \\ Universidad Católica de Santiago de Guayaquil, Ecuador
}

\begin{abstract}
RESUMEN Durante los años noventa, se hallaba extendida en los círculos académicos y en la sociedad en general la idea de que internet se convertiría en el medio a través del cual la humanidad alcanzaría una verdadera intercomunicación democrática. No obstante, apenas inaugurado el nuevo siglo, el idealismo primigenio fue abandonado por una visión más escéptica, la cual denunciaba la eventual aparición de un mercado de datos personales y la disminución de la democracia cognitiva, como consecuencia de la personalización automática de contenidos. En ese sentido, el presente ensayo procura revitalizar el debate de los efectos negativos que genera la desregulación de los motores de búsqueda y redes sociales, especialmente en lo que refiere a la censura previa y el tráfico ilícito de información personal.
\end{abstract}

PALABRAS CLAVE Filtro burbuja, datos personales, censura previa, personalización, tráfico de datos.

ABSTRACT During the nineties, it was widespread in academic circles and society the idea that the internet would become the means through which humanity would achieve true democratic intercommunication. However, just as the new century was inaugurated, the primal idealism was soon abandoned by a more skeptical vision, which denounced the possible emergence of a personal data market and the decline in cognitive democracy as a result of the automatic customization of content. In this sense, this essay seeks to revitalize the debate of negativity generated by non-regulation of search engines and social networks, particularly with regards to prior censorship and illicit trafficking in personal information.

KEYWORDS Bubble filter, personal data, pre-censorship, personalization, data traffic. 


\section{Introducción}

Como suele suceder con los progresos tecnológicos, la aparición del elemento determinante para la personalización informática no se dio por un acto intencionado. De hecho, nadie habría podido imaginar que el requerimiento de un cliente de Netscape Communications relacionado a una aplicación de compras en línea llevaría a uno de los problemas técnicos más trascendentales de los noventa, cuya resolución terminaría desembocado en la aparición de las cookies. ${ }^{1}$

En realidad, poco le importaba al cliente de Netscape el dar paso a una de las mayores innovaciones informáticas del siglo XX. Lo que en realidad le interesaba era disminuir los costos producidos por el almacenamiento y registro de toda la información que generaban las transacciones de sus compradores en línea. En sus escasos conocimientos de programación, el cliente de Netscape se cuestionaba por qué no guardar la información de las transacciones en el propio procesador del usuario, con el fin de que el portal de compras solamente interactúe con ellos al momento de efectuarse la operación comercial, y así evitar un gasto innecesario en el almacenamiento de datos. Esta idea, contenida en la sencilla interrogante del dueño del portal de compras, fue la que, una vez transmitida al personal técnico de Netscape Communications, terminaría por revolucionar el mundo de la web, y daría paso a la era de la personalización digital.

Finalmente, como explica Rossi (2018), la cuestión terminaría siendo resuelta por un joven de apellido Montulli, enrolado en el Departamento de Desarrollo de Netscape. Para Montulli, la resolución del problema planteado no exigía mayores esfuerzos: propuso la implementación de un mecanismo de registro con una memoria funcional, capaz de recordar cada página web con que el usuario se hubiera contactado en el pasado, con el fin de informar de manera anticipada sus preferencias a todos los sitios web con que este se contactase posteriormente, lo que reducía el tiempo de búsqueda y personalizaba los contenidos. Esta herramienta pasó a ser conocida bajo el rótulo de cookies.

A partir de este pequeño gran invento de Montulli, los navegadores y los sitios webs comenzaron a recordar a los internautas: así, las cookies ofrecieron las huellas mnémicas con las que la web comenzó a construir a sus usuarios modelo. Esas escurridizas entidades, situadas en la conjunción entre cuenta de usuario, ordenador y browser, eran ahora conjeturadas. Que el navegador sepa el nombre de usuario antes de escribirlo, que no sea necesario loguearse cada vez que se ingresa a un sitio o que el buscador hable el idioma de cada usuario, son tan solo algunas de las posibilidades habilitadas por este desarrollo (Rossi, 2018: 267).

1. John Schwartz, «Giving web a memory cost its users privacy», The New York Times, 4 de septiembre de 2001, disponible en https://nyti.ms/3mzsH7o. 
Sin la funcionalidad de las cookies, hoy cada acción que un usuario llevase a cabo en la internet seguiría siendo analizada como un fenómeno separado y hermético, sin referencia histórica o causal. De esta manera, la habitual previsión con la que funcionan los motores de búsqueda y los niveles de personalización que tiene la publicidad en redes sociales no serían posibles sin estas «galletas» (Cobo y otros, 2005). ${ }^{2}$

Sin embargo, no pasó mucho tiempo para que el mercado, bajo una lógica de lucro, identificase en esta herramienta atributos que ni Montulli ni Netscape Communications pudieron prever. Fue así como la herramienta creada inicialmente para facilitar la navegación de los internautas pasó a convertirse en una suerte de «doble agente» que, además de servir al usuario, servía a los dueños de los portales visitados. En la actualidad, se acusa a las cookies de ser uno de los principales medios para el tráfico de información privada, la publicidad basura y el enclaustramiento informático.

Montulli no imaginó (¿cómo podría?) que una simple galleta, como le gustaba llamar a las cookies, se convertiría en el primer eslabón de una enrevesada cadena alimenticia digital donde la publicidad online, la venta de datos personales y las burbujas de filtro, se contarían entre las especies deformes de ese ecosistema (Rossi, 2018: 267).

Con esto - como se expondrá más adelante-, el aprovechamiento de la funcionalidad de las cookies por parte de grandes grupos corporativos ha hecho posible la progresiva instauración de un ecosistema digital hipersegmentado, en que los algoritmos se encargan de construir una suerte de «celdas de panales» que separan a cada usuario de internet. Pero, a diferencia de lo que sucede con los panales de la vida real, en el entorno digital el gregarismo no constituye un leitmotiv, siendo este lugar ocupado por el hermetismo.

Precisamente, este hermetismo informático, que ha traído consigo la hiperpersonalización de los medios digitales, ha hecho que los sitios más concurridos de internet, como los principales motores de búsqueda (Google, Bing, Yahoo, etcétera), actúen de una manera poco neutral, al punto que «las respuestas a las mismas preguntas en los buscadores no facilitan las mismas respuestas para todos los usuarios, no muestran una única realidad. Estas respuestas difieren en función del individuo» (Tornero, 2018: 52). Así, ha quedado por completo rezagada la idea de un internet democratizante con información objetiva y neutral, ${ }^{3}$ para dar paso a la era de los «filtros burbujas».

2. En palabras de Rossi (2018: 267): «Las cookies se pueden diferenciar según: el tiempo que permanecen activas: i) persistentes: es decir, que tienen una fecha de caducidad definida, a fin de sobrevivir a varias sesiones de navegación. Una vez cumplida esa fecha, los datos registrados desaparecen. ii) No persistentes: se eliminan cuando el navegador se cierra. El seguimiento que hacen del usuario: i) locales: solo registran las acciones de los usuarios dentro del sitio. ii) De terceros: registran los movimientos del usuario más allá de un sitio específico. Se usan generalmente con fines publicitarios».

3. En la Declaración de Principios de la Cumbre Mundial sobre la Sociedad de Unión Internacional de Telecomunicaciones, uno de los primeros documentos oficiales de Naciones Unidas sobre internet, 


\section{El filtro burbuja}

La discusión sobre la presencia de mecanismos de segmentación de audiencias que afectan la conducta y actividad de los usuarios de internet ha estado presente desde finales de la década de los noventa. No obstante, ha sido Eli Pariser (2017) quien recientemente popularizó el debate sobre este tema, a partir de su obra El filtro burbuja: Cómo la red decide lo que leemos y lo que pensamos. Con su trabajo, Pariser procura llamar a una toma de conciencia respecto de las consecuencias negativas de incorporar en los portales digitales algoritmos diseñados para registrar, recordar, predecir y reproducir sucesivamente las acciones de los internautas, a los cuales los identifica a través de la figura metafórica de «filtros burbujas».

Para Pariser, mediante estos algoritmos los propietarios de los portales web recrean e imitan los intereses declarados previamente por sus usuarios, con el objetivo de desarrollar un bucle informático que los encapsula en una repetición incesante de sus preferencias, al ofrecerles una navegación altamente "personalizada».

A prima facie, pareciera que la existencia de estos filtros no tiene consecuencias negativas para el usuario, en tanto que le ofrecen atajos hacia sus reales intereses, y sirven como un remedio frente a la información basura que abunda en internet:

Estamos abrumados por un torrente de información: 900.000 entradas de blogs, 50 millones de tuits, más de 60 millones de actualizaciones de estado de Facebook, y 210.000 millones de correos electrónicos circulan cada día por el éter electrónico. [...] De modo que, cuando los filtros personalizados tienden una mano, nos inclinamos a aceptarla. En teoría, pueden ayudarnos a dar con la información que necesitamos saber, ver o escuchar - con aquello que nos importa de verdad- entre [el] cúmulo de imágenes de gatitos, anuncios de viagra o videos musicales de bailes sobre cintas de correr (Pariser, 2017: 45).

Sin embargo, en la realidad, los costos sociales y jurídicos que generaría este «mundo digital ptolemaico», en el cual la información — cual sol— gira a nuestro alrededor, podrían ser muy altos.

Bajo esta lógica, en la parte social, la estandarización de bucles informativos en los portales web podría facilitar el enclaustramiento selectivo de los usuarios en función de variables económicas, financieras y publicitarias fijadas de manera unilateral en beneficio de los propietarios de los portales. Mientras que, en lo concerniente a lo jurídico, la preeminencia de filtros burbujas podría dar paso a la proliferación de

se exponían como deseos y compromisos comunes: «[El] construir una sociedad de la información centrada en la persona, integradora y orientada al desarrollo, en que todos puedan crear, consultar, utilizar y compartir la información y el conocimiento, para que las personas, las comunidades y los pueblos puedan emplear plenamente sus posibilidades en la promoción de su desarrollo sostenible y en la mejora de su calidad de vida». 
nuevos esquemas de censura previa impropia, dentro de los cuales la pluralidad de las fuentes de información sería restringida en favor de una línea comunicacional establecida por los sitios web (Zuckerman, 2013).

A partir de esto, surge como hipótesis el pensar que las corporaciones informáticas cada vez van a tener menos interés en exponer a sus usuarios a nuevas experiencias, datos e informaciones de fuentes no familiares. Pero, al contrario de lo que se puede llegar pensar, el motivo de estas medidas no estaría determinado únicamente por la construcción de ambientes "personalizados» para los usuarios, sino también por la construcción de audiencias selectivas para las corporaciones. En efecto, si los sitios concentran sus esfuerzos en ofrecer a los usuarios contenidos elegidos acorde a sus preferencias, se deduce, de forma lógica, que aquellos permanecerán mucho más tiempo enganchados en sus interfaces, y por tanto más tiempo expuestos a la publicidad y al marketing digital, lo que finalmente significará mayores ingresos por ventas de espacios publicitarios para los dueños de los portales. En consonancia con esta hipótesis, Alberto Tornero ha señalado:

[Las corporaciones informáticas,] como no puede ser de otra forma, tratan que la búsqueda - o navegación web-suponga, vía una magnífica experiencia de usuario, la optimización de sus inversiones. Lo cual es completamente normal si entendemos que las compañías mercantiles, incluidas las empresas de internet, tienen ánimo de lucro. Aunque no paguemos por ellas. Desde los inicios de la televisión, pasando por Amazon, Google o Facebook, el autor va dando ejemplos de cómo la acción de los usuarios ha sido clave para el desarrollo y la oportunidad de negocio de estas empresas (Tornero, 2018: 53).

Por estas razones, las observaciones de Pariser configuran un importante sustrato teórico para evaluar el rol pasivo que adopta el usuario durante la construcción de los ambientes digitales «personalizados». En especial, en lo que respecta al desconocimiento que, en la mayoría de los casos, el usuario tiene sobre las actividades que los algoritmos y las cookies van a realizar en su procesador, así como la falta de consentimiento para la recolección de sus datos personales para fines publicitarios. Esto evidencia un grave problema de unidireccionalidad configurativa. ${ }^{4}$

De hecho, diversos experimentos han demostrado que los principios de pluralidad informativa, objetividad y neutralidad de la internet han venido siendo desplazados para favorecer el «emburbujamiento» de los internautas. Prueba de lo dicho

4. Las sospechas que esta configuración unidireccional levanta se intensifican si se toma en cuenta el tipo de sujetos que llevan a cabo la tarea "personificativa». En efecto, si el servicio de personalización de los internautas fuese prestado por personas naturales o colectivos sin fines de lucro, el riesgo de que exista un trasfondo mercantilista sería menor. Empero, al ser un proceso ejecutado por varias de las compañías más ricas del mundo, el riesgo de que como trasfondo subyazca una simple dinámica de mercado se eleva. 
se desprende del experimento llevado a cabo por Pariser, en el que solicitó a dos mujeres buscar un mismo término en el motor de Google, cada una desde su computador personal; comprobándose que el explorador arrojaba, en cada caso, resultados cuantitativa y cualitativamente diferentes. Lo mismo sucedería cuando Rossi, en el contexto latinoamericano, evidenciara la tendencia englobante del buscador de Google, en oposición al carácter genérico y neutral de buscadores sin hipermediación algorítmica. Resulta cada vez más evidente la presencia de una relación directamente proporcional entre el fin de lucro de algunas empresas como Facebook y Google y el enclaustramiento informativo de sus usuarios (Tornero, 2018: 53).

Esta relación proporcionalmente directa entre: i) la familiaridad del contenido; ii) el tiempo de exposición de los usuarios; y iii) la oportunidad de venta de publicidad u otros servicios podría suponer la aparición de una nueva máxima de la economía de plataformas, mediante la cual se afirmaría que a mayor familiaridad de contenidos (Pi), mayores serán los ingresos de las digital factories ( $\mathrm{Ij})$.

No obstante, el meollo de la problemática está lejos de agotarse en la aparición de una nueva dinámica económica, puesto que el emburbujamiento de los usuarios induciría al nacimiento de un sesgo cognitivo con características generalizantes y de extensión progresiva. En fin, la población inmersa en internet terminaría por ser encerrada en segmentos herméticos prefijados, cuyos individuos solo pueden interactuar con otros que compartan sus mismos gustos, ideologías e intereses (Nyhan y Reifler 2010). Esto es, estos procesos de enclaustramiento selectivo, filtros burbujas y bucles de información generarían un caldo propicio para el cultivo de nuevas realidades que impactan el ámbito regulatorio del derecho informático, de las cuales, por la magnitud de sus repercusiones, destacan el tráfico ilícito de datos personales y la censura previa.

\section{El mercado de datos personales}

Las «Big Five», nombre con el que se conoce al grupo de compañías conformado por Google, Amazon, Facebook, Apple y Microsoft, irrumpieron en el mercado de internet durante el período de transición de los siglos XX y XXI, como una opción fresca frente a los esquemas tradicionales. Por medio de la moderna codificación de sus softwares, originalmente estas compañías impulsaron la generalización de funciones innovadoras en los portales digitales y el posicionamiento de los usuarios en roles activos de edición, publicación y retroalimentación. Producto de esto, la web del siglo XXI dejó de verse como un gran archivero reservado para círculos gubernamentales, académicos y corporativos, y empezó a manifestarse como una verdadera comunidad virtual, libre de cualquier barrera física o de infraestructura. Con esto nació la internet 2.0 (De Bustos y Moreno Cano, 2018).

5. Antes de la aparición de la web 2.0, el principal medio de tráfico de datos en internet eran los co- 
Gillespie (2010: 350) define a la red 2.0 como un medio en el cual es posible materializar la intercomunicación en línea con sustento «en la habilidad de las personas para usar una serie de tecnologías de información y comunicación ofrecidas por una plataforma, para expresarse y participar en el espacio común generado por el ciberespacio». Casos como los de Wikipedia, el de las redes sociales o los buscadores modernos constituyen muestras paradigmáticas de este tipo de ambiente digital, en que los sitios web dejan de representar fuentes estáticas de información y se convierten en escenarios de diálogo y debate:

De este modo, el término plataforma - propio de la web 2.0- conlleva connotaciones que califican a los entornos producidos por los proveedores de servicios en línea como abiertos, neutrales, igualitarios y progresivos. Un entorno que empodera a sus usuarios a hablar y participar en el discurso democrático, enfatizando el contenido generado por el público, la creatividad popular, el contacto social entre pares y el comentario robusto (Álvaro, 2018: 77).

Sin embargo, como un efecto colateral, la aparición de la red 2.o. dejó al descubierto el potencial económico de un mercado gobernado por los datos informáticos. Esto llevó a que muchas compañías informáticas, entre estas las Big Five, desplegaran en sus portales y programas informáticos una serie de redes algorítmicas con la capacidad de registrar, almacenar y clasificar sistemáticamente datos personales, ${ }^{6}$ asegurándose el tener la información necesaria «sobre la percepción de una audiencia acerca de determinados temas». De ahí que se haya buscado acaparar datos «geográficos, demográficos, sicográficos y de personalidad», por medio de los cuales se pudiese saber «casi todo sobre ti: dónde vives, qué compras [...] y, aún más importante, si estás dispuesto a votar o si puedes ser persuadido para votar por determinado candidato» (Villalobos, 2018: 33).

Ciertamente, la potencialidad y novedad económica del big data —este cúmulo de datos personales ordenados de manera sistemática- y sus implicaciones dentro de la esfera del neuromarketing hacen de su apropiación un bien muy preciado para cualquier operador de mercado inmerso en un mundo globalizado, en el que las comunicaciones y transacciones se ejecutan mediante internet. En particular en el ám-

rreos electrónicos.

6. Como ha señalado Pariser (2017: 80): «El interés económico ha llevado a estas empresas a acumular cada vez más datos, mediante la adquisición de nuevos servicios —en el caso de Google, mediante la creación de Gmail en 2004, Blogger en 2003, Picasa en 2004, servicios cartográficos y de localización geográfica con la adquisición de Keyhole Inc. en 2004 y la posterior creación de Google Maps. Por su parte, Facebook adquirió Messenger en 2014, servicio de mensajería instantánea que se uniría a Whatsapp y a la red social basada en fotografías Instagram - o la prestación de servicios a terceros, botones de like o compartir y servicios de login que se distribuyen en la red creando un ecosistema conectado, que permite a estas corporaciones rastrear las acciones de sus usuarios a través de la web». 
bito publicitario, el big data constituye una herramienta eficaz para el aumento en el nivel de la certidumbre y la efectividad de impacto a la hora de transmitir un mensaje publicitario.

Para una mejor compresión del impacto del big data en la publicidad, téngase en cuenta que si un auspiciante publicitario contrata una campaña en medios de comunicación convencionales (radio, televisión), el mensaje publicitario será proyectado a todo tipo de público; en cambio, si lo hace empleando las utilidades de la hiperpersonalización digital y el embuclamiento informático, dicho mensaje solo será transmitido a una audiencia singularizada. En consecuencia, mientras que el grado de efectividad de las campañas publicitarias clásicas reside en la repetición y exposición constante de un mensaje, en los modelos publicitarios que funcionan a partir de big data la efectividad reside en la personalización digital.

Sobre este tema, Álvaro, quien a su vez cita a Gillespie (2014), reconoce al menos seis dimensiones negativas que ocupa el fenómeno algorítmico de captación de datos personales en la era de big data, en la medida en que dichos algoritmos eventualmente afectarían la construcción política, social, y económica de cada individuo sentado frente a una pantalla, en pos de la maximización de la utilidad publicitaria de las plataformas web:

Los procesos de colección y formalización de datos crean patrones de inclusión, que determinan qué es susceptible de ser un contenido computable y por tanto ser distribuido por la plataforma. También crean ciclos de anticipación, sometiendo a sus usuarios a procesos de vigilancia y extrayendo conclusiones a partir de categorías parciales y pobremente definidas. Categorías como edad, situación, familiar, tendencias políticas, aficiones, nivel cultural, ocupación profesional, se definen a partir de los datos generados en nuestras interacciones, como los dispositivos y lugares desde los que nos conectamos, amigos con los que interactuamos o contenidos que compartimos. Estas son empleadas en la definición de un perfil que ayuda a los anunciantes a saber cuáles son nuestros hábitos y preferencias, si podemos estar interesados en sus productos y si somos objetivos deseables. Una tercera dimensión de los algoritmos evalúa la relevancia de los contenidos en base a criterios de eficiencia y premisas epistemológicas ocultas. Estas acciones operan bajo una cuarta dimensión, la premisa de objetividad, que mantiene estos procesos al margen de procesos de enjuiciamiento, mientras se implican en la práctica y producen públicos calculados, últimas dos dimensiones. Estas dimensiones implican los algoritmos en la emergencia de procesos y agentes que median el discurso público y modifican el entorno comunicativo, poniéndolo a disposición no solo de estrategias de promoción y venta, sino de la manipulación política. Agentes maliciosos se ven capacitados para crear mensajes a medida y destinados a ciertos grupos para promover o desacreditar líderes políticos o instigar el odio hacia determinadas minorías (Álvaro, 2018: 78). 
A partir de esto, puede evidenciarse que el esquema clásico de personalización informativa - diseñado para que, en el tiempo de navegación web del usuario, las plataformas recolecten información valiéndose de cookies y algoritmos para luego, con la información recopilada, ser capaces de predecir y reproducir lo que el usuario requiere en cada sesión activa $-{ }^{7}$ ha sido reemplazado por un neoesquema de personalización, en el cual la información explorada por el usuario no se identificará por completo con el contenido que la página reproduzca, como resultado de la precarización y el sesgo de las variables y herramientas de recolección de datos. En este sentido, como ha señalado Álvaro (2018: 78-80), al momento del registro y reproducción predictiva de la información del usuario, las plataformas digitales estarían considerando los requerimientos de las compañías que contratan sus espacios publicitarios, tanto o más que las propias preferencias de los internautas.

Así, en el neoesquema, entre los distintos contenidos que el usuario haya visitado en sus exploraciones previas, se preferirá reproducir aquellos que guarden una mayor consonancia con el producto o servicio para el cual los servicios de publicidad de la plataforma han sido contratados. Por citar un ejemplo, imagínese un usuario N, cuyos intereses informativos se limitan a dos objetos en específicos de igual preferencia: el fútbol y el básquetbol. Bajo el esquema inicial de personalización, el contenido a replicarse se limitaría a reproducir en idénticas proporciones contenido sobre fútbol y básquet. No obstante, si a esto se le suma la presencia de una compañía con interés en promocionar en dicha plataforma su merchandising sobre fútbol, va a ser mucho más probable que la replicación que se efectúe, so pretexto de personalización, desconozca la proporción inicial (50\% futbol y 50\% básquet), y favorezca a la línea de contenido que mayor afinidad guarde con el producto publicitario de la contratante (por ejemplo, $75 \%$ de fútbol y $25 \%$ de básquetbol).

Con esta evolución, la función de las plataformas digitales hiperpersonalizadas no se limitaría a reproducir los contenidos captados antes (idea clásica de bucle informativo), sino que expondrían una preferencia subjetiva en la reproducción de aquella información que le reportase mayores réditos económicos en materia de publicidad. Por lo tanto, la idea inicial de configuración de audiencias selectivas mediante discriminación de datos mutaría a la de creación de audiencias por influencia en la información, o sea, las plataformas habrían pasado de identificar audiencias a crear tendencias (Cancela, 2019).

Sin embargo, el esquema antedicho no sería el único método de aprovechamiento de datos personales por parte de los portales digitales. Un caso llamativo, por su alto grado de innovación y mimetismo tecnológico, se observa en aquel fenómeno que

7. Esto originaba una relación de identidad entre la información explorada de forma inicial por el usuario y la posteriormente reproducida; circunstancia que podría ser denominada como «efecto espejo», en que lo buscado al principio coincide con el contenido ofertado después. 
envuelve un tráfico directo de datos personales a través de aplicaciones, juegos, grupos y demás funcionalidades incluidas en las redes sociales, como lo verificado con Cambridge Analytica, un ejemplo paradigmático de este tipo de sucesos.

Así, en el caso Cambridge Analytica, los datos personales de miles usuarios de Facebook fueron captados por la corporación Global Science Research (GSR), una compañía de propiedad del sicólogo social Alexander Kogan, por medio de una plataforma de entretenimiento ofertada por Facebook. En concreto, GSR creó un cuestionario en línea llamado «Happiness \& kindness», el cual, bajo la apariencia de ser un examen sicológico gratuito, solicitó el consentimiento de 270.000 titulares de cuentas de Facebook para recopilar datos personales de ellos y luego comercializarlos (Cancela, 2019).

Como resultado, se comprobaría que durante el tiempo que funcionó, Cambridge Analytica habría logrado recopilar información de cerca de 87 millones de personas, cuyos datos fueron vendidos a grupos económicos y campañas electorales en Estados Unidos y Reino Unido. A su vez, se estima que Facebook habría sacado un importante provecho de la venta de los espacios donde funcionaban y se exponían las aplicaciones y publicidades de Cambridge Analytica.

Sobre el episodio, Vercelli (2018: 5) ha sido enfático al sentenciar que

en Cambridge Analytica no solo querían mostrar anuncios publicitarios dentro de Facebook Inc.: pretendían crear perfiles según rasgos similares (aproximados) de personalidad para enviar anuncios microsegmentados a los diferentes grupos de votantes (grupos de personalidad de los votantes según rasgos sicométricos). Así, buscaron emular el funcionamiento interno de Facebook Inc. (es decir, aquello que solo disponía la corporación puertas adentro) para hacerlo funcionar con nuevos algoritmos y por fuera de la plataforma.

A su vez, De Bustos y Moreno Cano (2018), citando a Zuckerman, han indicado que anomalías como las de Cambridge Analytica evidencian que el «pecado capital» del internet moderno nace por la penetración de dinámicas de hiperexposición publicitaria. Este pecado capital se manifestaría principalmente a través de cuatro puntos focales: i) el binomio publicidad y vigilancia; ii) la cultura del meme o viralización de contenido; iii) el centralismo web; y iv) los fenómenos de emburbujamiento digital.

En lo que concierne al binomio publicidad y vigilancia en las plataformas digitales, a diferencia de lo que sucede en el espionaje y la vigilancia estatal, la causa de la vigilancia no responde a una lógica de seguridad nacional, sino que es un antecedente necesario para el microtargeting. Para esto, las plataformas necesitan observar permanentemente a sus usuarios (tener acceso a su actuar en la red en cualquier momento), para así llegar a recopilar información relevante que, una vez sometida a ciertas variables, sirva para conformar audiencias segmentadas llamativas para ser ofertadas a compañías en busca de publicidad. 
Fuchs (2012: 147), a propósito de este punto, ha expresado que «estas plataformas se basan en la publicidad y acumulan ganancias por la "publicidad dirigida" que requiere la vigilancia masiva para la mercantilización de los datos personales del usuario e información relativa a su comportamiento con fines económicos».

Por su parte, en lo que corresponde a la viralización de contenidos, los sitios web se valen de ciertas características antropológicas del ser humano para atraer su atención y enganchar su tiempo. Para tales fines se utiliza, a modo de carnada, el equivalente de la comida chatarra en el campo de la información: la trash data, entre cuyos especímenes se cuentan un «cúmulo de imágenes de gatitos, anuncios de viagra y videos musicales de bailes sobre cintas de correr», como denuncia Pariser (2017: 52).

Danah Boyd (2009), sobre esto, ha manifestado:

Nuestro cuerpo está programado para consumir grasas y azúcares porque son raros en la naturaleza. [...] Del mismo modo, estamos biológicamente programados para prestar atención a las cosas que nos estimulan: a contenidos que son groseros, violentos o sexuales, a esos chismes que son humillantes, embarazosos y ofensivos. Si no tenemos cuidado, vamos a desarrollar el equivalente sicológico a la obesidad. Nos encontraremos consumiendo el contenido que menos nos beneficie, a nosotros o a la sociedad en general.

Paradójicamente, el problema de la viralización se sostiene gracias a los elementos digitales que más inofensivos pueden parecer: likes, views, retweets y shares. En definitiva, estos instrumentos, cuya presencia podría ser identificada como accidental y no sustancial, constituyen una vía principal y directa de conexión entre nosotros y el plano digital. Por lo tanto, cada vez que se oprime el botón de like en Facebook o se hace un retweet en Twitter, comunicamos a dichas plataformas nuestras preferencias, debilidades y deseos. Sin embargo, el conflicto de esta colección binaria de datos, con la utilización de emoticones y botones de interfaz (like/dislike, thumbs up/thumbs down, follow/unfollow, etcétera), no se reduce solo a la reproducción de un mundo sesgado en que prevalecen nuestros gustos; sino que, además, le da una preeminencia a material de entretenimiento (videos virales, memes, violencia, pornografía, etcétera), en desmedro de noticias de interés público. Una vez más, cabrá recordar que lo que se busca es repetir la ecuación antes citada, en que el mayor tiempo de exposición en la plataforma ocasionará mayores espacios publicitarios vendidos $\mathrm{y}$, por consiguiente, aumento en los ingresos (Cancela, 2019). ${ }^{8}$

8. «De este modo, la nueva "cultura del meme" imprimió una vuelta de tuerca más tanto al consumismo como a los rasgos tecnocráticos del neoliberalismo: el mercado no solo excluía la participación del individuo en la toma de decisiones que afectaban a su vida en sociedad en internet, o determinaba su identidad digital mediante motores de búsqueda o redes sociales, sino que la libertad de consumo, acompañada de una producción voraz de noticias, dio lugar a tanta bullshit que el usuario perdió el norte de lo que lo rodeaba con tanta rapidez como su condición de ciudadanos» (Cancela, 2019: 143). 
Por su lado, en lo que tocante a la centralización digital, es factible reparar en la presencia de una contradicción de fondo que subyace en la campaña de «personalización» impulsada por las corporaciones de software, en particular los motores de búsquedas y las redes sociales. Así, pues, al mismo tiempo que las compañías digitales postulan la atomización de los internautas, trabajan en forma paralela en pos de la masificación de sus portales. En consecuencia, se observa que, además de la postulada personalización digital, existe un desarrollo de estrategias para acaparar la mayor cantidad de usuarios en sus redes, lo que desnuda un trasfondo económico. De este modo, dichas empresas requieren del emburbujamiento informático de sus usuarios a fin de evaluar sicométricamente su vulnerabilidad publicitaria y captar datos específicos de preferencia. Empero, también requieren de millones de personas conectadas a su web para poder ofertar audiencias lo suficientemente grandes para competir con otros espacios publicitarios de recepción masiva, como la televisión y la radio. De un modo más laxo, lo que se procura es la atomicidad a nivel personal, pero la masificación desde un enfoque comercial.

A pesar de los discursos sobre la personalización de los anuncios, los anunciantes, todavía acostumbrados a la tradición de las grandes audiencias que se conseguían en la televisión, prefieren las grandes audiencias. Por un lado, Facebook busca ampliar a toda costa el número de usuarios, para así poder ofrecer más conocimiento de más personas y así poder vender más a los anunciantes. Para crecer a toda costa, una de las acciones más usuales es la de adquirir competidores posibles (Pariser, 2017: 56). ${ }^{9}$

Estos intentos de masificación son los que han llevado a las redes sociales a instaurar formularios mínimos para el registro de usuarios, los que generalmente carecen de herramientas de certificación. Así, no es para nada inusual observar usuarios conectados a Facebook que no cumplen con la edad mínima para tener una cuenta, y mucho menos para celebrar un contrato electrónico con el cual autoricen el empleo de cookies y el acceso a sus datos personales. Las hordas de trols y bots que invaden Twitter, Instagram y Youtube son otra evidencia de esta masificación indiscriminada, en la cual no se hace casi ningún esfuerzo para certificar la procedencia de los usuarios o, por lo menos, su existencia plena.

Para finalizar el análisis de los puntos focales de Zuckerman, el problema de las

9. «Debido a este suceso, las empresas publicitarias eligieron difundirse de manera mucho más visual y rentable a través de plataformas como Instagram o Youtube, dirigidas principalmente a un público joven que ya no consumía prensa. Y aunque ello tuviera mayor relación con las lógicas posmodernas, más susceptibles a que su atención fuera atrapada por las mercancías de consumo, también explicaba que, por cada dólar en publicidad digital, Google y Facebook ganaran 75 centavos. Este era el motivo por el que ambas controlaban el $54 \%$ del negocio de publicidad digital del mercado global, impulsando así vertiginosamente su valor en el mercado de valores mientras decaía la legitimidad de los periódicos» (Pariser, 2017: 95). 
fakes news y las burbujas de filtro debe ser estudiado como una fenomenología de implicación mutua. Lo dicho se explica por cuanto la sobreexplotación que sufre el eje de familiaridad y aceptación en los bucles informativos llega a niveles tales que se prefiere dar a los usuarios noticias falsas que le gusten antes que información real que les pueda llegar a molestar y alejar de sus computadores. No es casual que junto a la aparición de los algoritmos de burbujas se hayan esparcido con fuerza algunas narrativas seudocientíficas que parecían extintas hace solo diez años, como la flat-Earth theory (teoría de la Tierra plana) o la vaccine hesitancy, el movimiento antivacunas.

De todo lo expuesto, podría colegirse una línea evolutiva en los métodos de captación y aprovechamiento comercial de datos personales mediante el emburbujamiento de usuarios. En un primer momento tenemos una etapa de personalización pura, en que la explotación comercial de datos se efectuaba mediante la utilización de audiencias selectivas, formadas por los intereses y preferencias manifestados por los usuarios de la plataforma; para luego pasar a una fase de creación de tendencias, a través de una replicación no neutral de datos recopilados; para finalmente llegar al tráfico directo de datos personales mediante herramientas de entretenimiento de redes sociales y la hiperexposición publicitaria con proliferación de fake news.

No obstante, a diferencia de lo que se ha percatado en lo que respecta a la rápida evolución de los métodos para captación de datos personales, en el plano jurídico ha existido una verdadera involución sobre este tópico. En efecto, esta idea se colige por el hecho de que, bajo los esquemas recién citados, no se configura una relación contractual efectiva, esto es, cuyo contenido repose en un vínculo consensual, ni un correspondiente equilibrio económico. De hecho, el tráfico de datos personales ejecutado por las plataformas digitales, por lo general, se encuentra estructurado bajo características de tripartismo y asimetría informativa.

Para entender el carácter tripartito de este tipo de fenómenos, basta con fijarse en el hecho de que entre los dos sujetos principales de la interacción de datos - a saber, los generadores y propietarios de aquellos y sus explotadores comerciales- se encuentra posicionada una plataforma digital, la cual opera como un intermediario atípico, cuya función, a diferencia de lo que sucede comúnmente con los intermediarios mercantiles, no es la de conectar los intereses comerciales de estas dos partes, sino la de explotar la información de una y venderla a otra. Esto podría dar paso a un nuevo análisis jurídico del tráfico ilegitimo de datos personales desde la teoría civil del enriquecimiento sin causa, entendido lato sensu como el enriquecimiento que obtiene un sujeto a costa de otro sin que haya mediado una causa jurídica (Abeliuk, 1971 :153).

Bajo esta línea, si se asume la lógica del enriquecimiento sin causa, se denotaría que las plataformas digitales se están apropiando de un elemento inmaterial de naturaleza inalienable, es decir, los datos personales de sus usuarios, para transferirlos de manera onerosa directa (banco de datos) o indirectamente (segmentación de audiencias) a una tercera persona, sin que en este ejercicio de tráfico el titular de los 
datos obtenga algún tipo de ganancia por la transacción llevada a cabo. De ser así, los usuarios de los portales digitales, especialmente de las redes sociales, de conformidad con distintas legislaciones domésticas de naturaleza continental, eventualmente tendrían el derecho de ejercer una acción de repetición en contra de estas compañías.

Por último, en lo que se relaciona a la asimetría de información, si bien es cierto que en muchos de los casos se les pide a los usuarios su consentimiento de manera clara - o solapada, como sucedió con Cambridge Analytica- para el uso de sus datos personales, en la mayoría de estas situaciones los usuarios desconocen cuál será el fin último y específico en que estos serán empleados, ya que describe en general su empleo en campos como «publicidad», «investigación social», «análisis de datos», etcétera, sin que lleguen a conocer de forma específica los procesos mercantiles en los que sus datos personales serán utilizados y quiénes harán uso de aquellos (Cubillos Vélez, 2017: 34).

\section{Obstáculos jurídicos para la regulación del tráfico de datos y la hiperexposición publicitaria}

Pese al amplio espectro de consecuencias negativas que se le atribuyen a la hiperexposición publicitaria en plataformas digitales, hoy son múltiples los obstáculos que se presentan para erigir un proceso de regulación jurídica a tales situaciones. Sustentan lo dicho la falta de normativa y los conflictos de jurisdicción y territorialidad.

Con respecto a lo primero, se ha demostrado que, a consecuencia de la férrea defensa de los valores capitalistas en los Estados modernos, importantes proyectos normativos como la Consumer Privacy Bill of Rights Act, impulsada por Barack Obama en 2015, o la Customer Online Notification for Stopping Edge-provider Network Transgressions Act, surgida luego del escándalo de Cambridge Analytica, han sido fuertemente rechazados. Entre los principales motivos con los que se ha justificado este rechazo, se cita a una supuesta pérdida de competitividad; en efecto, se menciona que los altos costos de implementación de los ajustes técnicos que los mentados proyectos normativos postulan implicarían una desequilibrio en la eficiencia empresarial. ${ }^{10}$

Empero, incluso en escenarios geográficos con regulaciones rígidas, como Europa y Reino Unido, el principal inconveniente para la eficacia en la regulación del tráfico de datos personales se observa en la metaterritorialidad de los ilícitos digitales. Ciertamente, la metaterritorialidad de la información digital envuelve dos supuestos.

10. En el plano regional, un caso similar fue el proyecto de ley orgánica de la protección de los derechos a la intimidad y privacidad sobre los datos personales, que presentó la presidenta de la Asamblea del Ecuador, Gabriela Rivadeneira, en 2016, el cual nunca superó los debates necesarios para su aprobación, producto de las críticas de los medios de comunicación y los gremios comerciales. 
Por una parte, el tráfico de datos no necesariamente se puede entender como un fenómeno corpóreo. Aquello sucede por cuanto los millones de bits en los que viaja nuestra información utilizan como vía un escenario de impulsos energéticos sin que se afirme en forma fehaciente que tal ecosistema digital se corresponde con la base geográfica de algún Estado. A ello habrá que añadir la transnacionalidad de las principales compañías informáticas —en su mayoría asentadas en Estados Unidos-, mismas que, cobijadas por la falta de tipicidad de sus actuaciones en los Estados donde se domicilian, tienen asegurada la impunidad para sus ejecutivos y patrimonios.

Recientemente se han ensayado algunas soluciones a los conflictos provocados por la jurisdicción y territorialidad difusa. Destacan entre estas la ratio decidenci de la sentencia que el Tribunal de Justicia de la Unión Europea expidió el 13 de mayo de 2014, en el caso C-131/12, en el cual se determinó:

El artículo 4, apartado 1, letra a), de la Directiva 95/46 $6^{11}$ debe interpretarse en el sentido de que se lleva a cabo un tratamiento de datos personales en el marco de las actividades de un establecimiento del responsable de dicho tratamiento en territorio de un Estado miembro, en el sentido de dicha disposición, cuando el gestor de un motor de búsqueda crea en el Estado miembro una sucursal o una filial destinada a garantizar la promoción y la venta de espacios publicitarios propuestos por el mencionado motor y cuya actividad se dirige a los habitantes de este Estado miembro (TJUE, Sentencia de 13 de mayo de 2014, asunto C-131/12). ${ }^{12}$

En tal virtud, se erige como una regla jurisprudencial en Europa la fijación de responsabilidad solidaria de las subsidiarias con respecto a los actos contrarios al derecho que ejecutan sus matrices estadounidenses.

Pese a esto, se ve con preocupación la reticencia que han demostrado ciertos organismos a nivel doméstico con respecto a adoptar la regla jurisprudencial del Tribunal de Justicia de la Unión Europea. Recientes resoluciones judiciales de Estados miembros han intentado modificar lo definido por el tribunal europeo, a través de nuevos

11. Directiva 95/46/CE del Parlamento Europeo y del Consejo, de 24 de octubre de 1995, relativa a la protección de las personas físicas en lo que respecta al tratamiento de datos personales y a la libre circulación de estos datos: «Artículo 4 Derecho nacional aplicable 1. Los Estados miembros aplicarán las disposiciones nacionales que haya aprobado para la aplicación de la presente directiva a todo tratamiento de datos personales cuando: a) el tratamiento sea efectuado en el marco de las actividades de un establecimiento del responsable del tratamiento en el territorio del Estado miembro. Cuando el mismo responsable del tratamiento esté establecido en el territorio de varios Estados miembros deberá adoptar las medidas necesarias para garantizar que cada uno de dichos establecimientos cumple las obligaciones previstas por el derecho nacional aplicable».

12. Sentencia del caso Google Spain, S.L. y Google Inc. con Agencia Española de Protección de Datos (AEPD) y Mario Costeja González, Tribunal de Justicia (Gran Sala). asunto C-131/12, decisorio, punto 2, de 13 de mayo de 2014, disponible en bit.ly/3flqkCQ. 
argumentos, como lo ha hecho la Tercera Sala del Tribunal Supremo del Reino de España, en la sentencia del 14 de marzo de 2016, donde resolvió que

es Google Inc. quien gestiona el motor de búsqueda - Google Search-, sin que en ningún caso se evidencie participación alguna en ese cometido de Google Spain, S. L., cuya actividad es la propia de un establecimiento (filial o sucursal) de aquella que se limita a la promoción y venta en España de los espacios publicitarios del motor de búsqueda, y en este sentido constituye una actividad conexa o vinculada económicamente a la de su matriz, pero de distinta naturaleza a la determinación de fines o medios del tratamiento. $Y$ es que no debe identificarse ni confundirse la determinación de los fines y medios del tratamiento, que caracteriza la condición de responsable, con una actividad de colaboración en la consecución de sus objetivos, que en el Dictamen 1/2010 se identifica genéricamente como actividad de otros agentes. De ahí que solo Google Inc. es la responsable del tratamiento, pues a ella corresponde en exclusiva la determinación de los fines, las condiciones y los medios del tratamiento de datos personales. ${ }^{13}$

Pese a lo expuesto, según ha señalado Moreno Muñoz (2018), agotar el análisis del presente tópico a una perspectiva netamente regulatoria, constituiría un craso error:

Abordar el problema de su impacto social disruptivo exclusivamente bajo la perspectiva legal orientada a la protección de los consumidores y de la información sensible que generan apenas supone rozar la superficie del fenómeno. El comportamiento de Facebook ilustra a la perfección un modelo de negocio sustentado en la cesión de datos de millones de sus usuarios a terceros, abusando de las zonas grises del marco regulador o directamente al margen de este. Las dudas al respecto se disiparon tras las declaraciones de sus máximos responsables de estrategia corporativa en diversas comisiones del Congreso estadounidense y del Parlamento europeo, una vez conocido el escándalo de Cambridge Analytica.

Bajo este ánimo ilativo, reducir las alternativas resolutivas del conflicto a un dilema de fortalecimiento regulatorio e imputación subjetiva de sucursales suena bastante ingenuo. Mucho más si se considera la evolución exponencial de la informática, que en poco tiempo relegaría cualquier tipo de regulación que se ensaye; así como la imposibilidad del rastreo de la mayoría de los propietarios de los sitios web. Por consiguiente, lo más acertado sería procurar la construcción de mecanismos de empoderamiento democrático para los usuarios, los cuales les permitan ser conscientes de la fenomenología algorítmica que los acecha día a día, y tomar decisiones informadas, es decir, sin perjuicio de las regulaciones legislativas y jurisprudenciales que se originen, se debe optar por proteger de forma reforzada el derecho de los usuarios

13. Tribunal Supremo del Reino de España. Sentencia STS 574/2016, 14 de marzo de 2016. acápite séptimo. 
a conocer el trato que se le está dando a su información y datos, y la forma en que la internet «personaliza» su contenido. ${ }^{14}$

\section{El retroceso de la democracia cognitiva y la censura previa}

Los fenómenos de microsegmentación intencionada, conocidos como «filtros burbujas», traducen dos tipos de afecciones sobre la libertad de expresión. El primero, de carácter objetivo, surge con respecto a la construcción de ese «bucle de ti mismo», que propicia la lógica algorítmica de las plataformas. Con ello, se procura sobresaturar de información afín a nuestro pensamiento, lo que degenera en un encapsulamiento de datos ya conocidos. Esta aparente simpleza grafica como ultima ratio una profunda herida en la construcción comunicacional de las sociedades modernas, sobre todo en las aristas de la consecución de una democracia cognitiva, el libre acceso de los conocimientos y el diálogo racional (Tornero, 2018).

Por definición, un mundo construido sobre la base de lo que nos resulta familiar es un mundo en el que no hay nada que aprender. Si la personalización es demasiado específica, esta puede impedir que entremos en contacto con experiencias alucinantes y aniquiladoras de prejuicios, así como con ideas que cambien nuestra forma de pensar con respecto al mundo y a nosotros mismos (Pariser, 2017: 83).

El segundo punto controvertido es fruto del pensamiento mercantilista que persiguen las corporaciones privadas a cargo de las grandes plataformas web, por ejemplo, las Big Five. Al estar el determinismo algorítmico diseñado para proporcionarnos información previamente aprobada o calificada positivamente por nosotros, dicho determinismo tenderá a acercarnos a contenidos que guarden relación con el entretenimiento, la farándula o cualquier otro tipo de información banal que opere bajo la lógica de la viralización de contenido y cultura del meme. ${ }^{15} \mathrm{~A}$ la larga, lo que lo que se

14. Álvaro (2018: 80) ha manifestado lo mismo al decir: «La decadencia de la esfera pública es un hecho complejo cuya solución no puede dejarse solo en manos de los recursos tecnológicos de los gigantes tecnológicos que gobiernan internet, ni en una legislación impuesta desde arriba por instituciones alejadas de los hechos y las problemáticas diarias. Esta requiere de la participación de todos los implicados y del desarrollo de herramientas tecnológicas o tradicionales, pero siempre creativas, que ayuden a la comprensión y una participación activa en las mediaciones que conforman la representación y la acción en nuestro mundo».

15. «En última instancia, el filtro burbuja puede afectar a nuestra capacidad para elegir cómo queremos vivir. El catedrático Tochai Benkler sostiene que para ser autores de nuestra vida debemos ser conscientes de una amplia gama de opciones y estilos de vida. Cuando entramos en un filtro burbuja, permitimos que las empresas que lo construyen elijan opciones de las que no somos conscientes. Podemos pensar que somos capitanes de nuestro destino, cuando lo cierto es que la personalización puede conducirnos a un cierto tipo de determinismo informativo en el que aquello sobre lo que clicamos en el pasado determine lo que vayamos a ver después» (Pariser, 2017: 23). 
produce en el usuario es la desconexión de noticias que importen un interés público real. Mark Zuckerberg, en defensa de estos procesos, ha llegado a afirmar que «saber que una ardilla muere delante de tu casa en este momento puede ser más relevante para tu interés que el hecho de que la gente se muera en África». ${ }^{16}$

Este último tópico, a diferencia del primero, vulnera de forma directa los intereses individuales de los usuarios, no solo a la sociedad en general. La privación intencionada de contenido de interés público constituiría un caso de censura previa atípica. Bajo esta modalidad, no serían los Gobiernos, sino corporaciones con un fuerte poderío económico las que decidirían qué contenido puedes o no observar según sus intereses particulares. Lo expuesto se entiende mejor si se advierte que la «personalización» efectuada por Facebook, Apple o Google contiene en su base la presencia de un interés de lucro, de modo tal que el emburbujamiento, antes que beneficiar el confort informático de sus usuarios, responde a la construcción de audiencias influenciables para sus clientes publicitarios.

Retomando el análisis de las consecuencias adversas que el emburbujamiento algorítmico eventualmente podría provocar sobre la democracia cognitiva, podemos empezar señalando que dicho concepto estructura una idea de democracia que, además de postular el acceso universal y sin discriminación a la información, defiende la necesidad del conflicto. En palabras de Riera (2009: 1), esta noción de democracia

supone el conflicto, porque comprende a las mayorías y las minorías de una sociedad, con sus respectivos intereses e ideologías, pero también requiere voluntad para superar las dificultades, atendiendo a resolver las necesidades y reclamos de los ciudadanos, porque la democracia es frágil y puede ser ahogada por los conflictos; por lo tanto, no se debe justificar un estado permanente de crispación que desencadene en reacciones que seamos incapaces de moderar. Se deben explorar las maneras de crear las condiciones bajo las cuales la violencia y la hostilidad inherentes a las relaciones sociales puedan ser desactivadas y desviadas para hacer posible un marco de convivencia.

En este sentido, el enclaustramiento informático ha dado pie a justamente lo contrario. Además de la proliferación de audiencias selectivas, ha favorecido al esparcimiento de grupos polarizados que cultivan todo tipo de discursos herméticos. De esta forma, en los mencionados grupos, las únicas noticias que circulan son aquellas que alimentan la polarización e inhiben la conquista de consensos democráticos. Como Álvaro (2018: 79) ha criticado:

Hoy en día esta esfera pública agoniza bajo el monopolio de las grandes compañías de medios y de la administración algorítmica que gobierna la comunicación

16. Eli Pariser, «When the internet thinks it knows you», The New York Times, 23 de mayo de 2011, disponible en nyti.ms/3wScApC. 

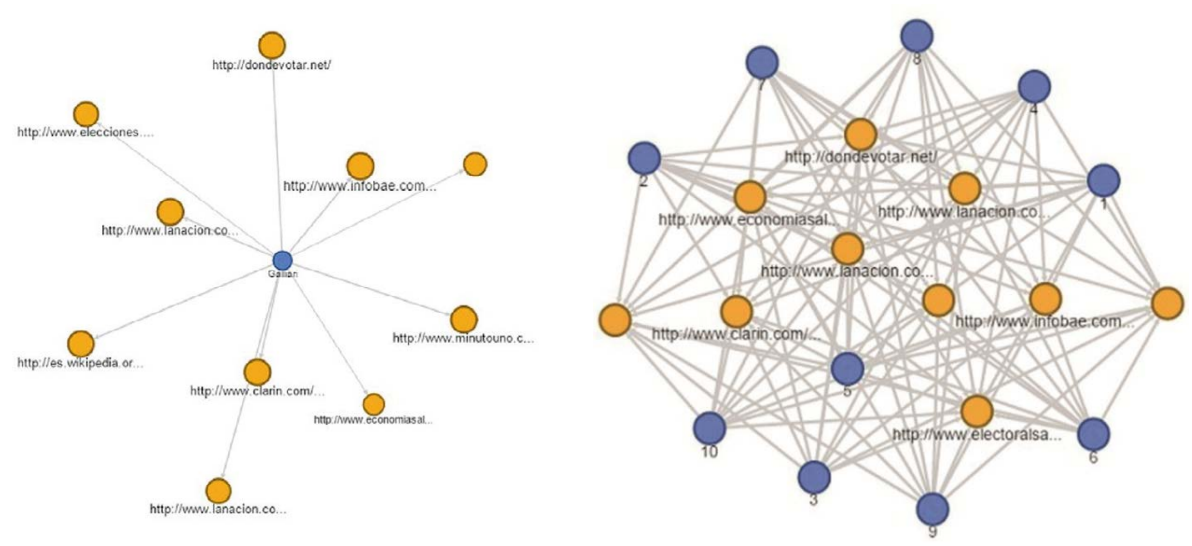

Figura 1. Diagramas de enclaustramiento informático según la neutralidad del buscador. Fuente: Rossi, 2018: 274.

en red. Si anteriormente la web había puesto al alcance de todos las herramientas para acceder y contribuir a un conocimiento producido y clasificado colectivamente, hoy en día, nuestras comunicaciones son procesadas, monitorizadas, clasificadas y distribuidas por nuevos agentes inteligentes que operan en un entorno complejo, un nuevo ecosistema que ha puesto en comunicación diversos agentes con distintos propósitos.

Pruebas científicas como las llevadas a cabo por Rossi en 2015 (figura 1) ${ }^{17}$ han comprobado que los buscadores genéricos, es decir, aquellos que carecen de algoritmos de emburbujamiento, tienden a ofrecer información con fuentes más nutridas, al contrario de lo que pasa con el motor de búsqueda de Google, el cual tiene cierta predilección por determinadas direcciones (URL) y tiende al enclaustramiento casi total de sus usuarios. Luego, si bien Rossi no ha exteriorizado los motivos por los que Google da preferencia a unas direcciones sobre otras, se podría colegir que se debe a razones netamente comerciales.

Fenómenos como estos, además de ser problemáticos en una esfera sociológica, también trastocan en forma importante a las esferas normativas. Junto con machacar varios de los fundamentos que inspiran la idea de Estado de derecho - marco de las sociedades modernas- y a la sociedad de la información, el enclaustramiento informativo vulnera los derechos sociales recogidos en catálogos dogmáticos de distintos cuerpos normativos.

Por ejemplo, en el caso ecuatoriano, su Carta Magna ha previsto de forma expresa

17. El primer diagrama de tipo estrella recrea la forma ideal en la que un buscador no neutral enclaustra a un usuario (punto más oscuro). En el segundo diagrama la relación usuario-dato se invierte, lo que evidencia una operabilidad genérica (Rossi, 2018: 274). 
en su artículo 16.1 el derecho de todas las personas a acceder a «una comunicación libre, intercultural, incluyente, diversa y participativa, en todos los ámbitos de la interacción social, por cualquier medio y forma, en su propia lengua y con sus propios símbolos». La noción de un bucle de noticias seleccionadas de forma unidireccional por un sistema de algoritmos no guardaría consonancia con los precitados principios de interculturalidad, diversidad y participación.

Igual nocividad traduciría para el derecho ecuatoriano aquella suerte de censura previa, analizada en líneas anteriores, puesto que el artículo 18.1 de la Carta Magna del Ecuador ha reconocido que «toda la persona, en forma individual o colectiva, tienen derecho a: 1) Buscar, recibir, intercambiar, producir y difundir información veraz, verificada, oportuna, contextualizada, plural, sin censura previa acerca de los hechos, acontecimientos y procesos de interés general, y con responsabilidad ulterior». Una fórmula normativa más precisa respecto de este tipo de censura previa atípica era la que contenía la antigua Ley Orgánica de Comunicación de la República de Ecuador, cuyo artículo 18 establecía que «la omisión deliberada y recurrente de la difusión de temas de interés público constituye un acto de censura previa».

Pero no solo la normativa ecuatoriana podría llegar a ser infringida mediante la fenomenología algorítmica de las grandes corporaciones digitales, por cuanto incluso aquella podría llegar a afectar al propio sistema interamericano de derechos humanos. Así, se puede observar cómo el contenido y el espíritu de textos jurídicos como la Convención Americana de Derechos Humanos y la Declaración de Principios sobre la Libertad de Expresión estarían siendo vulnerados. En lo relativo a este último cuerpo normativo, se puede esgrimir la trasgresión de su artículo 5, que, al igual que la normativa ecuatoriana, califica como censura previa cualquier restricción «en la circulación libre de ideas y opiniones, como así también la imposición arbitraria de información y la creación de obstáculos al libre flujo informativo, violan el derecho a la libertad de expresión». En definitiva, el texto expuesto recrea el concepto de filtro burbuja, tanto en el aspecto de restricción u obstáculo de flujo informativo, en tanto sirve como un tamiz selectivo de contenido para los usuarios; como en la imposición de cierta información específica, en cuanto a que, como señala Álvaro (2018: 75):

La web está sometida a la mediación de algoritmos que filtran y recomponen nuestra percepción del mundo al margen de nuestra consciencia. Un hecho que empeora cuando estos agentes son oscurecidos bajo las leyes de propiedad intelectual que protegen los intereses de los gigantes tecnológicos que han monopolizado la web, y cuando el último principio que rige estos procesos de extracción de datos y clasificación es la maximización del beneficio económico de un modelo basado en la economía de la atención y en el que el producto son los usuarios.

De hecho, en la misma línea, el Pacto de San José, en su artículo 13.3, ha sentenciado: 
No se puede restringir el derecho de expresión por vías o medios indirectos, tales como el abuso de controles oficiales o particulares de papel para periódicos, de frecuencias radioeléctricas, o de enseres y aparatos usados en la difusión de información o por cualesquiera otros medios encaminados a impedir la comunicación y la circulación de ideas y opiniones.

La Corte Interamericana entiende esta bidimensionalidad de la prohibición de censura previa y el respeto de la liberta de expresión, el cual, en sus palabras:

Incluye el derecho de expresar pensamientos e ideas y el derecho de recibirlas. Por lo tanto, cuando este derecho es restringido a través de una interferencia arbitraria, afecta no solo el derecho individual de expresar información e ideas, sino también el derecho de la comunidad en general de recibir todo tipo de información y opiniones. ${ }^{18}$

De manera precisa, esta hiperpersonalización de contenidos terminaría por vulnerar los principios de pluralidad y diversidad que el sistema interamericano de derechos humanos ha definido como los pilares del derecho de libertad de expresión, ya que el embuclamiento de usuarios restringiría tanto a la diversidad de fuentes de información (outlet) como de contenidos (output), limitándolas conforme a sus preferencias económicas de índole publicitaria (Lovatón Palacios, 2014: 73). ${ }^{19}$

\section{Dos alternativas a manera de conclusión}

En cuanto internet fue concebido como una herramienta para la consecución de la sociedad de la información, se vio en este un instrumento útil para procurar una formación integral de la sociedad. Esta herramienta se pensó como un escenario germinador de colisiones ideológicas que deriven en consensos políticos, económicos y científicos, todo lo cual despertaría el progreso social. Por ende, el deber primordial de la World Wide Web, por sobre erigirse como medio de difusión alternativo, era el de crear ciudadanos activos, que respondan eficientemente a las demandas sociales, con autonomía, solidaridad, responsabilidad y trascendencia, a través de una plataforma comunicativa. En otras palabras, internet nació como un catalizador de una democracia cognitiva digital.

18. Corte Interamericana de Derechos Humanos, Opinión Consultiva OC-5/85, 13 de noviembre de 1985, disponible en bit.ly/2Qm5qcl.

19. En 2007 , la declaración conjunta del relator especial de Naciones Unidas para la libertad de opinión y expresión, el representante de la OSCE para la libertad de los medios de comunicación, el relator especial de la OEA para la libertad de expresión y la relatora especial de la Comisión Africana sobre los Derechos Humanos y de los Pueblos determinó que «la diversidad en los medios es de fundamental importancia para la libre circulación de información e ideas en la sociedad [...] la diversidad tiene una naturaleza compleja, que incluye diversidad del outlet (tipos de medios) y de la fuente (propiedad de los medios), así como también diversidad de contenido (media output)», disponible en bit.ly/2RK76xA. 
Sin embargo, debido a la interacción de un sistema capitalista global y la hipermediación publicitaria que trajo consigo la posmodernidad, el internet ha mutado progresivamente hacia una suerte de herramienta sofisticada subordinada a los fines del mercado. Muestras de lo anterior son la censura previa y el uso de filtros burbujas, con sus correspondientes implicaciones en el tráfico de datos, en los motores de búsqueda y las redes sociales. La ineficiencia de los Gobiernos para resolver el problema de la desregulación de la internet demuestra que la primera alternativa para solventar el conflicto nace del empoderamiento de los usuarios. Como ha señalado Tornero (2018: 53):

El propio individuo tiene cierta responsabilidad en manejar su información, de modo que trate de minimizar posibles usos que puedan tener consecuencias no deseadas. Así, la aceptación de condiciones generales y políticas de privacidad, ya no sin leerlas, sino sin siquiera ojearlas, o la percepción de que, si algo es gratis, no me planteo qué puede buscar quien me proporciona esos servicios, sorprendentemente sin cobrar por ellos, son formas de actuar y pensar que el individuo debe replantearse.

Se vuelve, entonces, necesario que el usuario se encuentre informado de esta problemática, para que pueda tener cierto grado de control sobre sus datos personales y considere los riesgos a los que se expone dentro de las burbujas de contenidos desplegadas en las redes sociales y motores de búsqueda. Caso contrario, los seres humanos del presente siglo se verán expuestos a ceder la construcción de su propio pensamiento a un conjunto de números y fórmulas inertes que yacen en los códigos fuente de los principales sitios de internet. En consecuencia, el antiguo temor de autómatas laboralistas, recreado por el cine mudo alemán de principios del siglo XX, podría manifestarse en la actualidad bajo la figura de autómatas consumidores, que ceden su libertad de elección a cambio de entretenimiento.

Por otra parte, la segunda alternativa, de carácter más regulatorio, estaría direccionada hacia los órganos de los Gobiernos, en especial los encargados de las políticas legislativas y regulatorias. Los actuales esfuerzos que pretenden normar a las compañías de software, inspirados en ideas de comercio justo y defensa del consumidor, pese a acertar en su contenido axiológico, han sido ineficaces con respecto a su consecución procedimental. De ahí que una posible salida estaría en dejar de observar el problema jurídico de fondo bajo la lógica de una relación comerciante-consumidor, y empezar a estudiarlo desde la óptica de un vínculo de conocimiento-conocedor y de enriquecimiento justificado. En este sentido, a diferencia de lo que pasaría con una relación de consumo, en una relación cognitiva la finalidad está en la tutela no de un balance jurídico, sino de un balance informativo y económico. Con esta lógica, se podría exigir el enriquecimiento de las variables que consideran los algoritmos de los portales, a fin de no caer en los lugares comunes de entretenimiento y contenido 
relevante que inspiran sitios como Facebook, Instagram y Twitter. Sobre esto, Rossi (2018: 266) ha dicho:

No solo la idea de relevancia, sino, también, las de inconformidad, contenido estimulante e importante deberían ser parte de la ecuación. De igual manera, el análisis de lo que muestran y lo que dejan fuera los sistemas algorítmicos, los criterios a partir de los cuales trabajan, la información que disponen de los usuarios y el modo en que operan, resultan cuestiones que no pueden quedar ajenas a las discusiones sobre la ecología mediática actual.

Quizás lo más importante de esta última relectura planteada sería la vuelta al centro del conocimiento como una herramienta de perfeccionamiento individual y social, a fin de disminuir la brecha entre los países desarrollados y los que están en vías de desarrollo. De esa manera, podría volverse a encontrar el norte planteado por los primeros teóricos de la sociedad de la información, pues, como se ha señalado en demasía, las sociedades de consumidores estarán condenadas a ser gobernadas por las sociedades de los conocedores.

Luego, queda en nosotros, los internautas, la elección del papel que pretendemos ocupar en la historia.

\section{Referencias}

Abeliuk, René (1971). Las obligaciones. Santiago: Ediar.

Álvaro, Sandra (2018). «La esfera pública en la era de la hipermediación algorítmica, noticias falsas, desinformación y la mercantilización de la conducta». Hipertext, 17: 74-82. DOI: 10.31009/hipertext.net.2018.117.07.

Boyd, Danah (2009). «Streams of content, limited attention: The flow of information through social media». Conferencia WEB 2.o Expo, Nueva York, 17 de noviembre.

Cancela, Ekaitz (2019). Despertar del sueño tecnológico: Crónica sobre la derrota de la democracia frente al capital. Madrid: Akal.

Сово, Ángel, Patricia Gómez, Daniel Pérez y Rocío Rocha (2005). PHP y MySQL: Tecnologías para el desarrollo de aplicaciones web. Madrid: Díaz de Santos.

Cubillos Vélez, Ángela (2017). «La explotación de los datos personales por los gigantes de internet». Estudios en Derecho de la Información, 3: 27-55. DOI: 10.22201/ iij.25940082e.2017.3.10823.

De Bustos, Juan Carlos y Toñy Moreno Cano (2018). «Los señores de los datos: Google-Alphabet, Amazon, Facebook, Apple y Microsoft». Boletín de Recursos de Información, 53: 1-12. Disponible en https://bit.ly/3teDaqJ.

Fuchs, Christian (2012). «The political economy of privacy on Facebook». Television \& New Media, 13 (2): 139-159. DOI: 10.1177/1527476411415699. 
GiLlespie, Tarleton (2010). «The politics of "platforms"». New Media \& Society, 12 (3): 347-364. DOI: $10.1177 / 1461444809342738$.

-. (2014). «The relevance of algorithms». En Tarleton Gillespie, Pablo J. Boczkowski y Kirsten A. Foot (editores), Media technologies (pp. 170-190). Cambridge: MIT Press.

Lovatón Palacios, David (2014). «El equilibrio interamericano entre pluralidad de información y concentración de medios». Derecho PUCP, 73: 131-153. DOI: 10.1880o/derechopucp.201402.005.

Moreno MuÑoz, Miguel (2018). «Virtualización del espacio público y concepto débil de privacidad: Lecciones del caso Facebook-Cambridge Analytica». Ensayos de Filosofía, 8. Disponible en https://bit.ly/3snktQJ.

Nyhan, Brendan y Jason Reifler (2010). «When corrections fail: The persistence of political misperceptions». Political Behavior, 32 (2): 303-330. DOI: 10.1007/ S11109-010-9112-2.

PARISER, Eli (2017). El filtro burbuja: Cómo la red decide lo que leemos y lo que pensamos. Barcelona: Taurus.

Riera, Elba (2009). La democracia cognitiva: Una tarea pendiente. Buenos Aires: Comunidad de Pensamiento Complejo.

Rossi, Aníbal (2018). «¿Burbujas de filtro? Hacia una fenomenología algorítmica». Inmediaciones de la Comunicación, 13 (1): 263-281. DOI: 10.18861/ic.2018.13.1.2836.

STroie, Iuliana Raluca (2016). «¿Es o no es Google Spain responsable del tratamiento de datos personales?». Revista CESCO de Derecho de Consumo, 17. Disponible en https://bit.ly/zuQdykj.

Tornero, Alberto (2018). Tech \& Society: Un foro para pensar sobre el futuro de la sociedad tecnológica. Madrid: Fundación Telefónica.

VerCELLI, Ariel (2018). «La (des)protección de los datos personales: Análisis del caso Facebook Inc. y Cambridge Analytica». Conferencia en XVIII Simposio Argentino de Informática y Derecho (SID), Buenos Aires, 3 al 7 de septiembre.

VILlalobos, Valeria (2018). "Cambridge Analytica: De la interfaz al régimen». Revista de la Universidad de México, 5: 131-135. Disponible en https://bit.ly/3aaeQyZ.

Zuckerman, Ethan (2013). Rewire: Digital cosmopolitans in the age of connection. Nueva York: W. W. Norton \& Company.

\section{Sobre el autor}

Fernando Bajaña Tovar es abogado por la Universidad Católica de Santiago de Guayaquil, Ecuador. Asesor en Corte Constitucional del Ecuador. Su correo electrónico es fernando.bajana@cce.gob.ec. (D) https://orcid.org/oooo-ooo2-5348-3742. 


\title{
REVISTA CHILENA DE DERECHO Y TECNOLOGÍA
}

La Revista de Chilena de Derecho y Tecnología es una publicación académica semestral del Centro de Estudios en Derecho Informático de la Facultad de Derecho de la Universidad de Chile, que tiene por objeto difundir en la comunidad jurídica los elementos necesarios para analizar y comprender los alcances y efectos que el desarrollo tecnológico y cultural han producido en la sociedad, especialmente su impacto en la ciencia jurídica.

\author{
EDITOR GENERAL \\ Daniel Álvarez Valenzuela \\ (dalvarez@derecho.uchile.cl) \\ SITIO WEB \\ rchdt.uchile.cl \\ CORREO ELECTRÓNICO \\ rchdt@derecho.uchile.cl \\ LICENCIA DE ESTE ARTÍCULO \\ Creative Commons Atribución Compartir Igual 4.o Internacional
}

\begin{abstract}
La edición de textos, el diseño editorial
y la conversión a formatos electrónicos de este artículo

estuvieron a cargo de Tipográfica

(www.tipografica.io).
\end{abstract}

